\title{
NORMAL CURVATURE OF MINIMAL SUBMANIFOLDS IN A SPHERE
}

\author{
by SHARIEF DESHMUKH
}

(Received 23 June, 1995)

1. Introduction. Simons [5] has proved a pinching theorem for compact minimal submanifolds in a unit sphere, which led to an intrinsic rigidity result. Sakaki [4] improved this result of Simons for arbitrary codimension and has proved that if the scalar curvature $S$ of the minimal submanifold $M^{n}$ of $S^{n+p}$ satisfies

$$
\frac{n(n-1)\left(2 n^{2}+n-8\right)}{2\left(n^{2}+n-3\right)} \leq S
$$

then either $M^{n}$ is totally geodesic or $S=2 / 3$ in which case $n=2$ and $M^{2}$ is the Veronese surface in a totally geodesic 4-sphere. This result of Sakaki was further improved by Shen [6] but only for dimension $n=3$, where it is shown that if $S>4$, then $M^{3}$ is totally geodesic (cf. Theorem 3, p. 791).

Let $M^{n}$ be a compact minimal submanifold of the unit sphere $S^{n+p}$ with normal bundle $v$. We denote by $R^{\perp}$ the curvature tensor field corresponding to the normal connection $\nabla^{\perp}$ in the normal bundle $v$ of $M^{n}$, and define $K^{\perp}: M \rightarrow R$ by

$$
K^{\perp}=\sum_{i, \alpha, \beta}\left[R^{\perp}\left(e_{i}, e_{j}, N_{\alpha}, N_{\beta}\right)\right]^{2},
$$

where $\left\{e_{1}, \ldots, e_{n}\right\}$ is a local orthonormal frame on $M^{n}$ and $\left\{N_{1}, \ldots, N_{p}\right\}$ is a local field of orthonormal normals. We call the function $K^{\perp}$ the normal curvature of the minimal submanifold $M^{n}$. In this paper. we prove the following result.

THEOREM. Let $M^{n}$ be a compact minimal submanifold of $S^{n+p}$. If the normal curvature $K^{\perp}$, the scalar curvature $S$ and the square of the length of the second fundamental form $\sigma$ of $M^{n}$ satisfy

$$
K^{\perp} \leq \sigma, \quad S>(n-1)^{2},
$$

then $M^{n}$ is totally geodesic.

This theorem can be considered as a partial generalization of the result of Shen [6, Theorem 3]. However, it will be an interesting question whether the condition $K^{\perp} \leq \sigma$ is redundant and Shen's result can be extended beyond dimension 3 .

2. Preliminaries. Let $M$ be a minimal submanifold of the unit sphere $S^{n+p}$, with normal bundle $v$. Then the second fundamental form $h$ of $M^{n}$ satisfies

$$
(\nabla h)(X, Y, Z)=(\nabla h)(Y, Z, X)=(\nabla h)(Z, X, W), \quad X, Y, Z \in \mathscr{X}(M),
$$

where $\mathscr{L}(M)$ is the Lie algebra of smooth vector fields on $M$ and $(\nabla h)(X, Y, Z)$ is defined by

$$
(\nabla h)(X, Y, Z)=\nabla_{X}^{1} h(Y, Z)-h\left(\nabla_{X} Y, Z\right)-h\left(Y, \nabla_{X} Z\right),
$$

where $\nabla^{\perp}$ is the connection defined in $v$ and $\nabla$ is the induced Riemannian connection

Glasgow Math. J. 39 (1997) 29-33. 
with respect to the induced Riemannian metric $g$ on $M^{n}$. The second covariant derivative $\left(\nabla^{2} h\right)(X, Y, Z, W)$ of the second fundamental form is given by

$$
\begin{aligned}
\left(\nabla^{2} h\right)(X, Y, Z, W)= & \nabla_{X}^{\frac{1}{X}}(\nabla h)(Y, Z, W)-(\nabla h)\left(\nabla_{X} Y, Z, W\right) \\
& -(\nabla h)\left(Y, \nabla_{X} Z, W\right)-(\nabla h)\left(Y, Z, \nabla_{X} W\right), \quad X, Y, Z, W \in \mathscr{X}(M) .
\end{aligned}
$$

We have the following form of the Ricci identity

$$
\begin{array}{r}
\left(\nabla^{2} h\right)(X, Y, Z, W)-\left(\nabla^{2} h\right)(Y, X, Z, W)=R^{\perp}(X, Y) h(Z, W)-h(R(X, Y) Z, W) \\
-h(Z, R(X, Y) W), \quad X, Y, Z, W \in \mathscr{X}(M),
\end{array}
$$

where $R^{\perp}$ and $R$ are the curvature tensors of the connections $\nabla^{\perp}$ and $\nabla$ respectively. Since $M^{n}$ is a minimal submanifold for a local orthonormal frame $\left\{e_{1}, \ldots, e_{n}\right\}$ of $M^{n}$ we have

$$
\begin{aligned}
\sum_{i=1}^{n}(\nabla h)\left(X, e_{i}, e_{i}\right) & =0, \\
\sum_{i=1}^{n}\left(\nabla^{2} h\right)\left(X, Y, e_{i}, e_{i}\right) & =0 .
\end{aligned}
$$

Using the Ricci tensor Ric, we define the symmetric operator $R^{*}$ by

$$
\operatorname{Ric}(X, Y)=g\left(R^{*}(X), Y\right), X, Y \in \mathscr{X}(M) .
$$

Then the Gauss equation gives

$$
\begin{gathered}
\mathrm{A}_{h(Y, Z)} X=R(X, Y) Z+A_{h(X, Z)} Y-g(Y, Z) X+g(X, Z) Y, \\
R^{*}(X)=(n-1) X-\sum_{i=1}^{n} A_{h\left(e_{i}, X\right) e_{i}}, \quad X, Y, Z \in \mathscr{Z}(M),
\end{gathered}
$$

where $A_{N}, N \in v$, is the Weingarten map with respect to the normal $N$, satisfying $g\left(A_{N} X, Y\right)=g(h(X, Y), N)$. We define

$$
\begin{aligned}
\sigma & =\sum_{i, j}\left\|h\left(e_{i}, e_{j}\right)\right\|^{2}, \\
\left\|A_{h}\right\|^{2} & =\sum_{i, j, k}\left\|A_{h\left(e_{i}, e_{j}\right)} e_{k}\right\|^{2}, \\
\|\nabla h\|^{2} & =\sum_{i, j, k}\left\|(\nabla h)\left(e_{i}, e_{j}, e_{k}\right)\right\|^{2} .
\end{aligned}
$$

Now we prove the following lemma.

Lemma. Let $M^{n}$ be a minimal submanifold of $S^{n+p}$, then for a local orthonormal frame $\left\{e_{1}, \ldots, e_{n}\right\}$, we have

$$
\sum_{i, j, k} R\left(e_{k}, e_{i} ; e_{j}, A_{h\left(e_{i}, e_{j}\right)} e_{k}\right)=-\sigma+\left\|A_{h}\right\|^{2}+\frac{1}{2} K^{\perp}-\sum_{i, j, \alpha} g\left(A_{\alpha} e_{i}, A_{\beta} e_{j}\right)^{2}
$$

where $A_{\alpha} \equiv A_{N_{\alpha}}$ and $\left\{N_{1}, \ldots, N_{p}\right\}$ is a local field of orthonormal normals.

Proof. Using the Ricci equation

$$
R^{\perp}\left(X, Y ; N_{1}, N_{2}\right)=g\left(\left[A_{N_{1}}, A_{N_{2}}\right](X), Y\right), \quad X, Y \in \mathscr{Z}(M), N_{1}, N_{2} \in \nu,
$$


we get

$$
\begin{aligned}
K^{\perp} & =\sum_{i, j, \beta}\left[R^{\perp}\left(e_{i}, e_{j} ; N_{\alpha}, N_{\beta}\right)\right]^{2}=\sum_{i, j, \alpha, \beta}\left[g\left(A_{\alpha} A_{\beta} e_{i}, e_{j}\right)-g\left(A_{\beta} A_{\alpha} e_{i}, e_{j}\right)\right]^{2} \\
& =2 \sum_{i, j, \alpha, \beta} g\left(A_{\alpha} e_{i}, A_{\beta} e_{j}\right)^{2}-2 \sum_{i j, \alpha, \beta} g\left(A_{\alpha} A_{\beta} e_{i}, e_{j}\right) g\left(A_{\beta} A_{\alpha} e_{i}, e_{j}\right),
\end{aligned}
$$

since $\sum_{i, \alpha, \beta} g\left(A_{\alpha} e_{j}, A_{\beta} e_{i}\right)^{2}=\sum_{i, \alpha, \beta} g\left(A_{\beta} e_{j}, A_{\alpha} e_{i}\right)^{2}$ which follows from the symmetry of $A_{\alpha}$ and $A_{\beta}$. Next using the Gauss equation, we have

$$
\begin{aligned}
R\left(e_{k}, e_{i} ; e_{j}, A_{h\left(e_{i}, e_{j}\right)} e_{k}\right)= & \delta_{i j} g\left(h\left(e_{k}, e_{k}\right), h\left(e_{i}, e_{j}\right)\right)-\delta_{k j} g\left(h\left(e_{i}, e_{j}\right), h\left(e_{i}, e_{k}\right)\right) \\
& +g\left(h\left(e_{i}, e_{j}\right), h\left(e_{k}, A_{h\left(e_{i}, e_{j}\right)} e_{k}\right)\right)-g\left(h\left(e_{k}, e_{j}\right), h\left(e_{i}, A_{\left.h\left(e_{i}, e_{j}\right)\right)} e_{k}\right)\right)
\end{aligned}
$$

since $A_{h\left(e_{i}, e_{j}\right)} e_{k}=\sum_{\alpha} g\left(A_{\alpha} e_{i}, e_{j}\right) A_{\alpha} e_{k}$, we obtain

$$
\sum_{i, j, k} g\left(h\left(e_{i}, e_{j}\right), h\left(e_{k}, A_{h\left(e_{i}, e_{j}\right)} e_{k}\right)\right)=\sum_{i, j, k} g\left(A_{h\left(e_{i}, e_{j}\right)} e_{k}, A_{h\left(e_{i}, e_{j}\right)} e_{k}\right)=\left\|A_{h}\right\|^{2}
$$

and

$$
\sum_{i j, k} g\left(h\left(e_{k}, e_{j}\right), h\left(e_{i}, A_{h\left(e_{i}, e_{j}\right)} e_{k}\right)\right)=\sum_{i j, \alpha, \beta} g\left(A_{\alpha} A_{\beta} e_{i}, e_{j}\right) g\left(A_{\beta} A_{\alpha} e_{i}, e_{j}\right)
$$

Then using (2.7), (2.9) and (2.10) in (2.8) and using minimality of $M^{n}$ we find

$$
\sum_{i, j, k} R\left(e_{k}, e_{i} ; e_{j}, A_{h\left(e_{i}, e_{j}\right)} e_{k}\right)=-\sigma+\left\|A_{h}\right\|^{2}-\sum_{i, j, \alpha, \beta} g\left(A_{\alpha} e_{i}, A_{\beta} e_{j}\right)^{2}+\frac{1}{2} K^{\perp}
$$

which proves the lemma.

3. Proof of the theorem. Let $M^{n}$ be a compact minimal submanifold of $S^{n+p}$ satisfying the hypothesis of the theorem. Define $F: M \rightarrow R$ by $F=\frac{1}{2} \sigma$. Then it is straightforward to compute the Laplacian $\Delta F$ of the function $F$ as

$$
\Delta F=\sum_{i j, k} g\left(\left(\nabla^{2} h\right)\left(e_{k}, e_{k}, e_{i}, e_{j}\right), h\left(e_{i}, e_{j}\right)\right)+\sum_{i, j, k}\left\|(\nabla h)\left(e_{i}, e_{j}, e_{k}\right)\right\|^{2} .
$$

Using the Ricci identity (2.2) and equations (2.1) in above equation we arrive at

$$
\begin{aligned}
\Delta F= & \sum_{i, k}\left[R^{\perp}\left(e_{k}, e_{i} ; h\left(e_{k}, e_{j}\right), h\left(e_{i}, e_{j}\right)\right)-R\left(e_{k}, e_{i} ; e_{k}, A_{h\left(e_{j}, e_{i}\right)} e_{j}\right)\right. \\
& \left.-R\left(e_{k}, e_{i} ; e_{j}, A_{h\left(e_{i}, e_{j}\right)} e_{k}\right)\right]+\|\nabla h\|^{2} .
\end{aligned}
$$

We employ (2.4) in the Ricci equation, to compute

$$
\begin{aligned}
R^{\perp}\left(e_{k}, e_{i} ; h\left(e_{k}, e_{j}\right), h\left(e_{i}, e_{j}\right)\right)= & g\left(A_{h\left(e_{i}, e_{j}\right)} e_{k}, A_{h\left(e_{i}, e_{j}\right)} e_{k}\right. \\
& \left.+R\left(e_{i}, e_{k}\right) e_{j}-\delta_{k j} e_{i}+\delta_{i j} e_{k}\right)-g\left(A_{h\left(e_{k}, e_{j}\right)} e_{k}, A_{h\left(e_{i}, e,\right)} e_{i}\right)
\end{aligned}
$$

or

$$
\begin{aligned}
\sum_{i, j, k} R^{\perp}\left(e_{k}, e_{i} ; h\left(e_{k}, e_{j}\right), h\left(e_{i}, e_{j}\right)\right)= & \left\|A_{h}\right\|^{2}-\sigma+\sum_{i j, k} R\left(e_{i}, e_{k} ; e_{j}, A_{h\left(e_{i}, e_{j}\right)} e_{k}\right) \\
& -g\left(A_{h\left(e_{k}, e_{j}\right)} e_{k}, A_{h\left(e_{r}, e_{j}\right)} e_{i}\right) .
\end{aligned}
$$

Since (2.5) gives $R^{*}\left(e_{j}\right)=(n-1) e_{j}-\sum_{k} A_{h\left(e_{k}, e_{j}\right)} e_{k}$, we have

$$
\begin{aligned}
\sum_{i, j, k} g\left(A_{h\left(e_{k}, e_{j}\right)} e_{k}, A_{h\left(e_{i}, e_{j}\right)} e_{i}\right) & =\sum_{i, j} g\left((n-1) e_{j}-R^{*}\left(e_{j}\right), A_{h\left(e_{i}, e_{j}\right)} e_{i}\right) \\
& =(n-1) \sigma-\sum_{i, j} \operatorname{Ric}\left(e_{j}, A_{h\left(e_{i}, e_{j}\right)} e_{i}\right)
\end{aligned}
$$


Thus using (3.3) in (3.2), we have

$$
\begin{aligned}
& =(n-1) \sigma-\sum_{i, j, k} R\left(e_{k}, e_{j}, A_{h\left(e_{,}, e_{j}\right)} e_{i}, e_{k}\right) \\
& =(n-1) \sigma+\sum_{i, j, k} R\left(e_{k}, e_{i}, e_{k}, A_{h\left(e_{i}, e_{j}\right)} e_{j}\right) .
\end{aligned}
$$

$$
\begin{aligned}
\sum_{i, j, k} R^{\perp}\left(e_{k}, e_{i} ; h\left(e_{k}, e_{j}\right), h\left(e_{i}, e_{j}\right)\right)= & -n \sigma+\left\|A_{h}\right\|^{2}+\sum_{i j, k}\left[R\left(e_{i}, e_{k} ; e_{j}, A_{h\left(e_{i}, e_{j}\right)} e_{k}\right)\right. \\
& \left.-R\left(e_{k}, e_{i}, e_{k}, A_{h\left(e_{i}, e_{j}\right)} e_{j}\right)\right] .
\end{aligned}
$$

Using (3.4) in (3.1), we obtain

$$
\begin{aligned}
\Delta F= & -n \sigma+\left\|A_{h}\right\|^{2}-2 \sum_{i, j, k}\left[R\left(e_{k}, e_{i} ; e_{j}, A_{h\left(e_{i} \cdot e_{j}\right)} e_{k}\right)\right. \\
& -R\left(e_{k}, e_{i}, e_{k} ; A_{h\left(e_{i}, e_{j}\right)} e_{j}\right]+\|\nabla h\|^{2} .
\end{aligned}
$$

Also, we have

$$
\begin{aligned}
\sum_{i, j . k} R\left(e_{k}, e_{i} ; e_{k}, A_{h\left(e_{i}, e_{j}\right)} e_{j}\right) & =-\sum_{i, j} \operatorname{Ric}\left(e_{i}, A_{h\left(e_{,}, e_{j}\right)} e_{j}\right) \\
& =-\sum_{i, j} g\left(R^{*} e_{i}, A_{h\left(e_{i}, e_{j}\right)} e_{j}\right) \\
& =-\sum_{i, j, \alpha} g\left(R^{*} e_{i}, A_{\alpha} e_{j}\right) g\left(A_{\alpha} e_{i}, e_{j}\right) \\
& =-\sum_{i, j, \alpha} g\left(R^{*} A_{\alpha} e_{j}, e_{i}\right) g\left(A_{\alpha} e_{j}, e_{i}\right) \\
& =-\sum_{j, \alpha} g\left(R^{*} A_{\alpha} e_{j}, A_{\alpha} e_{j}\right) \\
& =-\sum_{j, \alpha} \operatorname{Ric}\left(A_{\alpha} e_{j}, A_{\alpha} e_{j}\right) \\
& =-\sum_{j, \alpha}(n-1) g\left(A_{\alpha} e_{j}, A_{\alpha} e_{j}\right)+\sum_{i, j, \alpha}\left\|h\left(e_{i}, A_{\alpha} e_{j}\right)\right\|^{2} \\
& =-(n-1) \sigma+\sum_{i j, \alpha, \beta} g\left(A_{\beta} e_{i}, A_{\alpha} e_{j}\right)^{2} .
\end{aligned}
$$

Using (3.6) and the lemma in Section 2 in (3.5), we obtain

$$
\Delta F=(n-1) \sigma-\left\|A_{h}\right\|^{2}+\left(\sigma-K^{\perp}\right)+\|\nabla h\|^{2} .
$$

Now using the facts that

$$
\begin{aligned}
\left\|A_{h}\right\|^{2} & =\sum_{i, j, k}\left\|A_{h\left(e_{i}, e_{j}\right)} e_{k}\right\|^{2}=\sum_{i j, k, \alpha} g\left(A_{\alpha} e_{i}, e_{j}\right)^{2}\left\|A_{\alpha} e_{k}\right\|^{2} \\
& =\sum_{i, j, \alpha} g\left(A_{\alpha} e_{i}, e_{j}\right)^{2}\left\|A_{\alpha}\right\|^{2}=\sum_{\alpha}\left\|A_{\alpha}\right\|^{2}\left\|A_{\alpha}\right\|^{2}=\sum_{\alpha}\left\|A_{\alpha}\right\|^{4}
\end{aligned}
$$

and $\sigma=\sum_{\alpha}\left\|A_{\alpha}\right\|^{2}$, in (3.7) and integrating it over $M^{n}$ we obtain

$$
\int_{M}\left\{\sum_{\alpha}\left[(n-1)-\left\|A_{\alpha}\right\|^{2}\right]\left\|A_{\alpha}\right\|^{2}+\left(\sigma-K^{\dot{1}}\right)+\|\nabla h\|^{2}\right\} d v=0
$$


From the hypothesis of the theorem $S>(n-1)^{2}$, it follows that

$$
n(n-1)-\sum_{\alpha}\left\|A_{\alpha}\right\|^{2}>(n-1)^{2},
$$

that is, $\sum_{\alpha}\left\|A_{\alpha}\right\|^{2}<(n-1)$, consequently $\left\|A_{\alpha}\right\|^{2}<(n-1)$, and that $K^{\perp} \leq \sigma$. Thus in order for (3.8) to hold we must have $\left\|A_{\alpha}\right\|=0$, that is $M^{n}$ is totally geodesic.

\section{REFERENCES}

1. J. L. Barbosa and M. do Carmo, Stability of minimal surfaces and eigenvalues of the Laplacian, Math. Z. 173 (1980), 13-28.

2. S.-Y. Cheng, Eigenvalue comparison theorems and its geometric applications, Math. Z. 143 (1975), 289-297.

3. S. S. Chern, M. do Carmo and S. Kobayashi, Minimal submanifolds of a sphere with second fundamental form of constant length, Functional analysis and related fields (Springer, 1970), 59-75.

4. M. Sakaki, Remarks on the rigidity and stability of minimal submanifolds, Proc. Amer. Math. Soc. 106 (1989), 793-795.

5. J. Simons, Minimal varieties in riemannian manifolds, Ann. of Math. (2) 88 (1968), 62-105.

6. Y. B. Shen, Curvature pinching for the three-dimensional minimal submanifolds in a sphere, Proc. Amer. Math. Soc. 115 (1992), 791-795.

Department of Mathematics

College of SCIENCE

King Saud University

P.O. Box 2455

RIYADH-11451

SAudi Arabia 